\title{
Grußwort des Bundesministers des Auswärtigen Heiko Maas
}

Die Verwirklichung der Deutschen Einheit am 3. Oktober 1990 markiert eine Zäsur, deren Wirkungen weit über die deutsche Geschichte hinausreichen und ohne die die heutige Verankerung unseres Landes in einem geeinten, friedlichen Europa und im multilateralen System undenkbar wäre. An diesem historischen Einschnitt ist nun auch die Edition der „Akten zur Auswärtigen Politik der Bundesrepublik Deutschland“ angelangt. Dies bietet die Gelegenheit zurückzublicken - auf 30 Jahre Deutsche Einheit und auf 30 Jahre Editionsgeschichte.

In der zweiten Hälfte der 1980er Jahre waren die wesentlichen Entscheidungen dafür getroffen worden, nach dem Abschluss der Reihe „Akten zur deutschen Auswärtigen Politik“ (1918-1945) nun auch ausgewählte Dokumente aus dem Politischen Archiv des Auswärtigen Amts zu den inter- und transnationalen Beziehungen der Bundesrepublik zu veröffentlichen. Zur Wahrung der wissenschaftlichen Unabhängigkeit wurde das renommierte Institut für Zeitgeschichte München-Berlin mit dieser Aufgabe betraut, das zu diesem Zweck eine eigene Editions-Abteilung zur Arbeit im Auswärtigen Amt ins Leben rief.

Am 1. Juni 1990 begannen die Arbeiten an drei Bänden zum Jahr 1963, die Ende 1993 veröffentlicht wurden. Seitdem wird jedes Jahr, stets nach Ablauf der dreiBigjährigen Aktensperrfrist, ein Jahrgang in zwei oder drei Teilbänden mit kommentierten Dokumenten der Öffentlichkeit zugänglich gemacht - eine Sammlung von mittlerweile über 13.000 erstmals veröffentlichten Dokumenten in 75 Bänden, die sich in den vergangenen 30 Jahren zu einem unverzichtbaren Referenzwerk für alle entwickelt hat, die sich für die Vorgeschichte unserer außenpolitischen Gegenwart interessieren.

Diese stolze Zahl gibt Anlass zu danken, allen voran den Herausgebern, von denen hier stellvertretend die drei Hauptherausgeber genannt werden sollen, die der Edition vorstanden bzw. noch vorstehen: Professor Dr. Dr. h.c. Hans-Peter Schwarz $(\dagger)$, Professor Dr. Dr. h.c. mult. Horst Möller und Professor Dr. Andreas Wirsching - sowie der Wissenschaftlichen Leiterin der Edition, Dr. Ilse Dorothee Pautsch, und ihrem Vorgänger Dr. Rainer A. Blasius. Auch allen anderen Wissenschaftlerinnen und Wissenschaftlern, die mit großem Einsatz an dieser Veröffentlichung mitgewirkt haben, sowie allen weiteren an diesem Werk Beteiligten spreche ich meinen Dank und meine Anerkennung für diese herausragende Leistung aus.

Nicht zuletzt gilt mein herzlicher Dank den Kolleginnen und Kollegen des Politischen Archivs im Auswärtigen Amt, ohne deren tatkräftige Unterstützung diese Arbeit nicht möglich wäre. In diesem Sinne wünsche ich der Edition der „Akten zur Auswärtigen Politik der Bundesrepublik Deutschland“ für die Zukunft weiterhin ein gutes Gelingen.

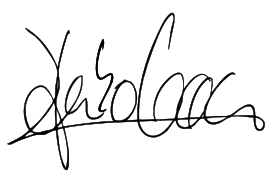


